\title{
A Practical Method to Predict the Incidence of Anterior Demodex Blepharitis
}

\author{
Yuan Heng Hsu ${ }^{1}$ and Wei Cherng $\mathrm{Hsu}^{* 2,3}$ \\ ${ }^{1}$ National Taiwan University College of Medicine, Taiwan \\ ${ }^{2}$ Department of Ophthalmology, Taipei Tzu Chi Hospital, Taiwan \\ ${ }^{3}$ Tzu Chi University College of Medicine, Taiwan
}

*Corresponding author: Wei Cherng Hsu, Tzu Chi Hospital, Buddhist Tzu Chi Medical Foundation, Office address: No. 289, Jianguo

Rd., Xindian Dist., New Taipei City 231, (R.O.C.) Taipei, Taiwan

\section{ARTICLE INFO}

Received: 㗀 January 27, 2019

Published: 㗀 February 01, 2019

Citation: Yuan Heng H, Wei Cherng H. A Practical Method to Predict the Incidence of Anterior Demodex Blepharitis. Biomed J Sci \& Tech Res 13(5)2019. BJSTR. MS.ID.002473.

Keywords: Anterior Demodex Blepharitis; Diagnosis

Abbreviations: FBS: Foreign Body Sensation; D. Folliculorum: Demodex. Folliculorum; D. Brevis: Demodex. Brevis
ABSTRACT

Most of the symptoms induced by allergens can be resolved by the use of medication, however the symptoms related to demodex can't be relieved with medicine. The symptoms of inflammation caused by Demodex can vary from none to very diverse and are often hard to cure with medical treatment. The purpose of this study is to find a way to increase the rate of diagnosis, and also to find connections between the four patient symptoms (Foreign body sensation, discharge, itching, and dryness) and the rate of infestation.

Material and Methods: When patients voluntarily mentioned one of the four symptoms in clinic, the doctor included them as a candidate in this study. The three epilated eyelashes from patients were examined using a slit lamp and a microscope (25 X, Olympus SZX16 model) for identification of demodex.

Results: The relationship between symptoms and positive infection rate is correlated.

Conclusion: Due to the inability of the anterior blepharitis diagnostics to isolate substances produced by the demodex, we constructed a method that provides doctors with a highly plausible clinical testing process.

\section{Introduction}

According to Medilexicon's medical dictionary, the prevalence rate of Allergic conjunctivitis is around $10 \%$. The causes include pollen, animal fur, contents of various eye drops, cosmetics, and dust mite tissue. Most of the symptoms can be resolved by the use of medication, however the symptoms of some patients can't be relieved with medicine. One of the reasons is that the reproduction of mites and the rate of allergen creation is not altered by medication and Blepharitis becomes a chronic decease. Demodex blepharitis generally results from $D$. folliculorum and $D$. brevis [1-3]. The former infests the roots of eyelashes, becoming the leading cause of anterior blepharitis. The latter infests the meibomian gland, causing posterior blepharitis. The symptoms of inflammation caused by Demodex can vary from none to very diverse and are often hard to cure with medical treatment. Damage caused by the D. folliculorum includes direct damage to the hair follicle and the introduction of the two bacterium (Streptococci and Staphylococci) which cause hyperplasia stratum corneum, resulting in the instability of the eyelash leading to an increase in the feeling of foreign body sensation (FBS).

The two bacteria may also have links to skin conditions like rosacea [4]. It can be assumed that waste from the demodex and allergic reactions will cause the amount of patient discharge to increase. The proteins and excreta created by the D. folliculorum can cause itchiness, the fourth type of Allergic immune response [2]. Inflammation of the general surface layer of the eye can result in instability in the number of tears and may cause subjective feelings of dryness. In conclusion, the clinical symptoms can be categorized into FBS, discharge, itching, and dryness. Potential criteria for diagnosis of Demodex blepharitis are the following: Clinical history, Slit-lamp examination, and microscopic confirmation. Even though the third method, microscopic confirmation, can directly prove the existence of the condition, we cannot guarantee its nonexistence 
on patients with negative results due to being unable to check every eyelash. The purpose of this study is to find a way to increase the rate of diagnosis utilizing the third method, and also to find connections between the four patient symptoms (FBS, discharge, itching, and dryness) and the rate of infestation.

\section{Material and Methods}

\section{Patient Population and Selection}

The study was conducted on 127 adult patients, composed of 29 males and 98 females, ranging from 58 to 96 years old, exhibiting at least one of the four symptoms.

\section{Methods of Data Collection}

When patients voluntarily mentioned one of the four symptoms in clinic, the doctor included them as a candidate in this study. The doctor recorded the symptoms of each individual patient assigning a score equally depending on the number of symptoms (score1 4 as blue bar in Figure 1) and epilated three random eyelashes from every study participant's upper eyelid on both sides. Sample preparation and identification of Demodex: The three epilated eyelashes from each participant's eyelid, were placed individually by order on slides with one drop of Normal saline and covered with a cover slip. The samples were examined using a slit lamp and a microscope (25 X, Olympus SZX16 model) Figure 2a \& 2b. All samples were evaluated within $10 \mathrm{~min}$ of removal from the eyelid. If Demodex was discovered, it was identified by its order and the sample number was recorded (Figure 3). If all three samples were clear of demodex, then it was recorded as non-infested.

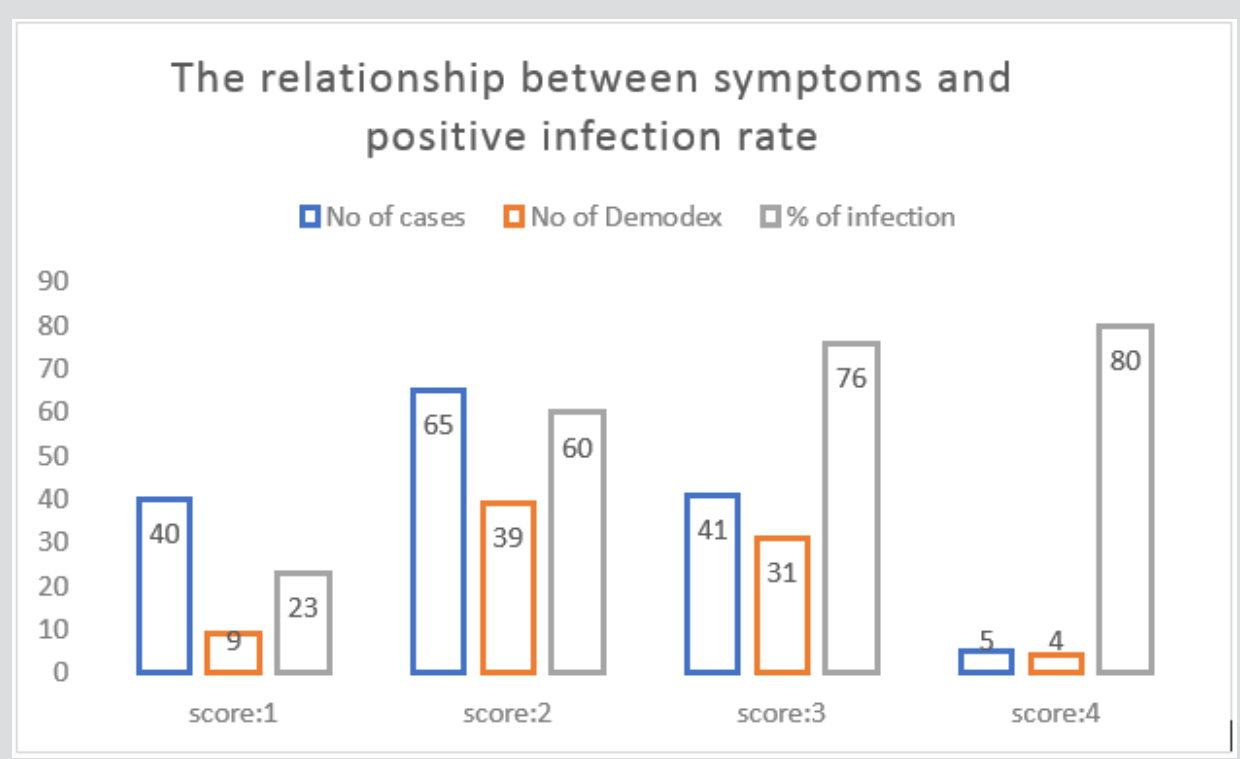

Figure 1: (The relationship between symptoms and positive infection rate: the blue bar represents the number of cases for each of the different score of symptoms. The red bar represents the number of positive demodex species found under microscopy in each group. The green bar represents the percentage of the red bar / blue bar (No. of cases with a positive finding in each group) / (No. of total cases in each group).

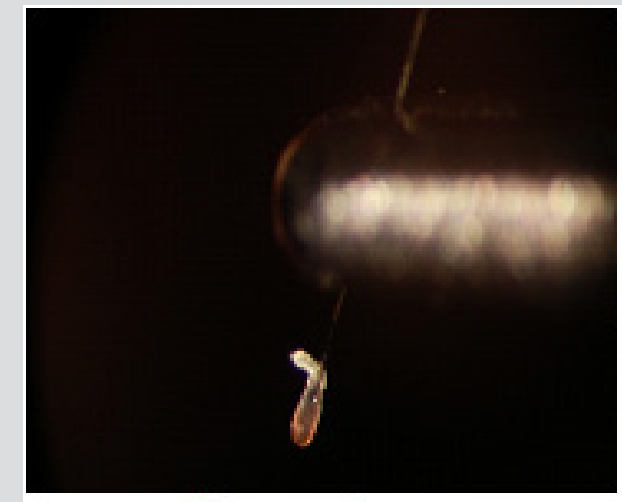

Figure 2a

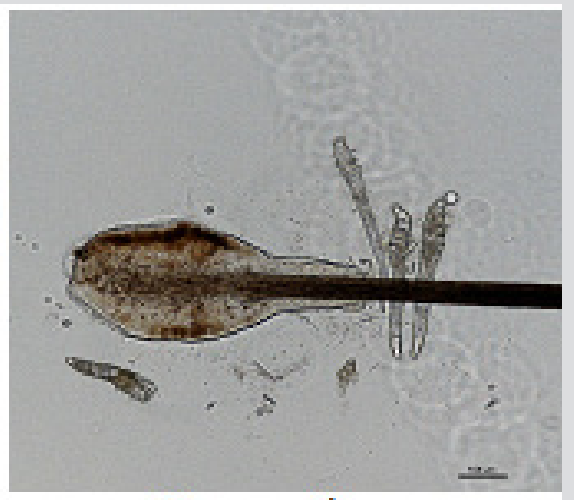

Figure $2 b$

Figure 2: Observation of the D. folliculorum's gathering around the hair follicle of an eyelash under slit lamp and microscopy (200 300Um). 


\section{The diagnostic proportion of three test eyelashes}

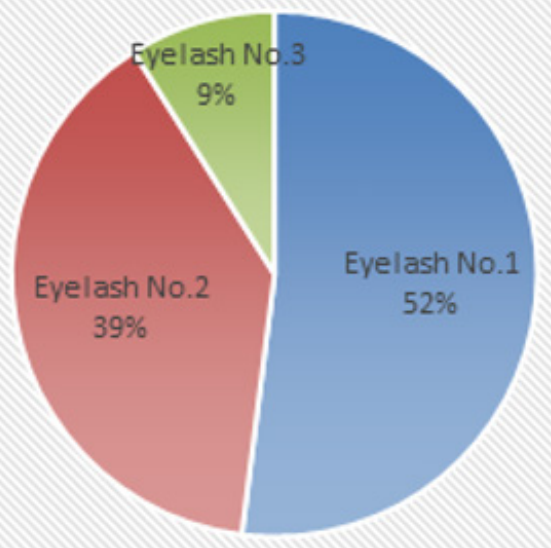

- Eyelash No.1

- Eyelash No. 2

m Eyelash No.3

Figure 3: The percent of Demodex found in the first and subsequent eyelashes is 52\% (Blue area), 39\%(Red area), and $9 \%($ Green area) in that order.

\section{Results}

The positive diagnosed rate is $67 \%$, with the average age of 72 years old (85/127). The clinical findings under slit lamp and microscopic findings are shown in figs.1a and $1 \mathrm{~b}$. The relationship between symptoms and positive infection rate is shown in Figure 1. The percent of Demodex found in the first and subsequent eyelashes is showed in Figure 3.

\section{Discussion}

Clinically, Demodex infestation can cause many ocular diseases, i.e. the destruction of eyelashes, Meibimitis leading to trichiasis or enlarged glands, a hypersensitive reaction inducing lid margin inflammation, an unstable tear film or even superficial corneal vascularization, marginal infiltration, a phlyctenule-like lesion, superficial opacity, and nodular scarring [5,6]. At present, detection and counting of Demodex eggs, larvae and adult mites in epilated lashes under microscopy can make the final diagnosis under Slit-lamp examination (dandruff at the root of eyelashes $[7,8]$ and clinical history (chronic blepharitis which is refractory to conventional treatments). However, a negative finding under microscopy cannot exclude the possibility of Demodex infestation. One limitation is that we can only see the D. folliculorum on top of the eyelash, and not those inside the sebaceous glands.

There is a strong correlation between a patient's serum reactivity to Bacillus antigen (a bacterium from Demodex 9,10,11) and the presence of ocular rosacea. This is indirect evidence for Demodex infestation $[9,10]$. Due to the inability of the anterior blepharitis diagnostics to isolate substances produced by the demodex, we constructed a method that provides doctors with a highly plausible clinical testing process. The results of the experiment show that there is a high positive correlation between the symptoms and infestation rate. It is also found that around $90 \%$ of the infestations can be identified through the first two eyelashes inspected, but there is still a chance of it not being accurate. If the patient's symptoms and signs are highly suspect for the diagnosis of demodex blepharitis, it is advisable to repeat the process to increase the diagnostic accuracy.

\section{Conclusion}

Demodex mite plays an important role in the occurrence of a series of ocular surface diseases such as Demodex blepharitis, meibomian gland dysfunction, conjunctival inflammation, and corneal lesions. Ocular infestation has a close relationship with the systemic infestation. Further studies are needed for developing easy and sensitive diagnostic methods and more effective and specific treating regimens.

\section{References}

1. Gao YY, Di Pascuale MA, Li W, Liu DT, Baradaran Rafii A, et al. (2005) High prevalence of ocular Demodex in eyelashes with cylindrical dandruffs. Invest Ophthalmol Vis Sci. 46(9): 3089-3094.

2. Bevins CL, Liu FT Rosacea (2007) Skin innate immunity gone awry? Nat Med 13(8): 904-906.

3. Gao YY, Di Pascuale MA, Elizondo A, Tseng SC (2007) Clinical treatment of ocular demodecosis by lid scrub with tea tree oil. Cornea 26(2): 136143.

4. Wolf R, Ophir J, Avigad J, Lengy J, Krakowski A (1988) The hair follicle mites (Demodex spp.). Could they be vectors of pathogenic microorganisms? Acta Derm Venereol 68(6): 535-537.

5. Gao YY, Di Pascuale MA, Elizondo A, Tseng SC (2007) Clinical treatment of ocular demodecosis by lid scrub with tea tree oil. Cornea 26(2): 136143. 
6. Kheirkhah A, Casas V, Li W, Raju VK, Tseng SC (2007) Corneal manifestations of ocular Demodex infestation. Am J Ophthalmol. 143(5): 743-749.

7. Gao YY, Di Pascuale MA, Li W, Liu DT, Baradaran-Rafii A, et al. (2005) High prevalence of ocular Demodex in eyelashes with cylindrical dandruffs. Invest Ophthalmol Vis Sci 46(9): 3089-3094.

8. Bevins CL, Liu FT (2007) Rosacea: skin innate immunity gone awry? Nat Med 13: 904-906.

\section{ISSN: 2574-1241}

DOI: 10.26717.BJSTR.2019.13.002473

Wei Cherng Hsu. Biomed J Sci \& Tech Res

(C) (i) This work is licensed under Creative

Submission Link: https://biomedres.us/submit-manuscript.php
9. Gupta AK, Chaudhry MM (2005) Rosacea and its management: an overview. J Eur Acad Dermatol Venereol 19(3): 273-285.

10. Li J, O’Reilly N, Sheha H, Katz R, Raju VK, et al. (2010) Correlation between ocular Demodex infestation and serum immunoreactivity to Bacillus microbial proteins in patients with facial rosacea. Ophthalmology 117(5): 870-877.

$\begin{array}{ll}\text { BIOMEDICAL } & \text { Assets of Publishing with us } \\ \text { RESEARCHES } & \text { - Global archiving of articles } \\ \text { - Immediate, unrestricted online access } & \text { - Rigorous Peer Review Process } \\ & \text { - Authors Retain Copyrights }\end{array}$

